\title{
Fibroblast activation protein- $\alpha$ in tumor cells promotes colorectal cancer angiogenesis via the Akt and ERK signaling pathways
}

\author{
FENG CAO $^{1}$, SONGSONG WANG $^{1}$, HUANQIN WANG $^{1}$ and WEI TANG ${ }^{2}$ \\ ${ }^{1}$ Department of Medicine 13, Xintai People's Hospital; ${ }^{2}$ Department of Anesthesiology, \\ 88 Hospital of PLA, Taian, Shandong 271000, P.R. China \\ Received January 12, 2017; Accepted September 6, 2017
}

DOI: $10.3892 / \mathrm{mmr} .2017 .8155$

\begin{abstract}
Fibroblast activation protein- $\alpha$ (FAP- $\alpha)$ is a cell surface serine protease of the post-prolyl peptidase family, and stromal FAP- $\alpha$ expression may serve important functions in tumor occurrence and progression. In recent years, FAP- $\alpha$ expression in tumor cells has been detected in a number of types of tumor, and its roles in tumor growth and metastasis have been reported. However, the presence of FAP- $\alpha$ in colorectal cancer (CRC) cells lacks sufficient evidence and its role in angiogenesis remains unknown. The present study confirmed FAP- $\alpha$ expression in CRC cells at the tissue and cellular level, using immunohistochemistry and western blot analysis, respectively; it additionally identified that FAP- $\alpha$ in CRC cells was positively associated with vascular endothelial growth factor (VEGF)-A expression and microvessel density in stained tissue samples for the first time. In addition, western blotting identified that FAP- $\alpha$ overexpression in SW1116 cells significantly upregulated VEGF-A expression, and silencing of FAP- $\alpha$ in HT29 cells markedly inhibited VEGF-A expression. Survival analysis demonstrated that patients with high expression of FAP- $\alpha$ and VEGF-A had the shortest survival time. To detect the effects of FAP- $\alpha$ on human umbilical vein endothelial cells (HUVECs), conditioned medium (CM) from $\mathrm{CRC}$ cell lines was used and it was identified that CM from SW1116 cells with overexpressed FAP- $\alpha$ exhibited significantly increased VEGF-R2, phosphorylated extracellular signal-regulated kinase ( $\mathrm{p}-\mathrm{ERK}$ ) and $\mathrm{p}-\mathrm{RAC}-\alpha$ serine/threonine-protein kinase (Akt) in HUVECs, in addition to the proliferation rate. Conversely, CM from HT29 cells with FAP- $\alpha$ silenced exhibited a significantly inhibited proliferation rate. Molecular mechanism analysis demonstrated that p-ERK and p-Akt in SW1116 and HT29 cells were affected by alterations in FAP- $\alpha$ expression, and treatment with a
\end{abstract}

Correspondence to: Dr Wei Tang, Department of Anesthesiology, 88 Hospital of PLA, 6 Hushan Road, Taian, Shandong 271000, P.R. China

E-mail: tangweitaian@163.com

Key words: fibroblast activation protein- $\alpha$, colorectal cancer, angiogenesis, microvessel density
p-ERK inhibitor (U0126) and p-Akt inhibitor (LY294002) ameliorated VEGF-A upregulation induced by FAP- $\alpha$ overexpression. All the results confirmed the presence of FAP- $\alpha$ in CRC cells and suggested that FAP- $\alpha$ may effectively promote angiogenesis in CRC via the Akt and ERK signaling pathways.

\section{Introduction}

Colorectal cancer (CRC) is one of the most common types of tumor worldwide with one of the highest mortality rates (1). Its occurrence is a complex process and may be caused by genetic or epigenetic alterations and different environmental factors (2). In recent years, with the development of early diagnosis and advanced systemic treatments, the overall prognosis has been ameliorated to a certain extent. However, for those with advanced and metastatic diseases, the prognosis remains poor $(3,4)$.

Angiogenesis, the formation of new blood vessels, may serve central roles in tumor occurrence and progression, and even prognosis (5). The newly-formed vessels faciliate nutrients and waste exchange for growing tumors, in addition to providing entry points for metastatic tumor cells (6). Finding effective targets which may significantly inhibit angiogenesis is likely to contribute to improved treatment outcomes for patients with cancer.

As a member of the serine protease family, an initial study reported that fibroblast activation protein- $\alpha$ (FAP- $\alpha$ ) was expressed in $>90 \%$ of epithelial tumor stromal cells and was able to serve important roles in proliferation, angiogenesis, immune escape, invasion and metastasis, and tissue remolding (7). Previous studies have demonstrated that FAP- $\alpha$ expression is not confined to stromal cells and is additionally expressed in certain malignant epithelial cells (8-10). Huang et al (8) reported that the human breast cancer cell line MDA-MB-231 expressing FAP- $\alpha$ grew more rapidly compared with a control group. Cheng et al (9) reported that HEK293 cells overexpressing FAP- $\alpha$ were $2-4$ times more likely to develop tumors in mice compared with HEK293 control cells, in addition to a 10- to 40-fold shift in tumor growth (9). In 2003, Iwasa et al (10) confirmed FAP- $\alpha$ expression in CRC cells using immunohistochemistry (IHC) staining and reported that high FAP- $\alpha$ expression was positively correlated with lymph node metastasis. However, the study lacked further 
cellular experiments and the specific function of FAP- $\alpha$ in $\mathrm{CRC}$ cells remains unknown. The present study focused on the proangiogenic roles of FAP- $\alpha$ derived from CRC cells, not mesenchymal cells, and further explored the potential mechanisms of angiogenesis regulated by FAP- $\alpha$.

\section{Materials and methods}

Cell lines and culture. Colorectal cancer cell lines SW1116 and HT29 and human umbilical vein endothelial cells (HUVECs) were all purchased from the American Type Culture Collection (ATCC; Manassas, VA, USA). SW1116 and HT29 cells were cultured in Dulbecco's modified Eagle's medium supplemented with $10 \%$ fetal bovine serum (FBS; both from Invitrogen; Thermo Fisher Scientific, Inc., Waltham, MA, USA). HUVECs were cultured in endothelial cell medium (Invitrogen; Thermo Fisher Scientific, Inc.) supplemented with 5\% FBS and 1\% endothelial cell growth supplement (Invitrogen; Thermo Fisher Scientific, Inc.). All cells were cultured at $37^{\circ} \mathrm{C}$ in $5 \% \mathrm{CO}_{2}$.

Tissue samples. With the approval from the Review Board and Ethics Committee of the People's Hospital of Xintai City, 80 primary CRC specimens were selected from patients who had undergone surgery between January 2006 and December 2014 in the Xintai People's Hospital (Shandong, China). No patients had received chemotherapy, radiotherapy or immunomodulatory therapy prior to surgery.

IHC. Sections from CRC samples were cut to $4-\mu \mathrm{m}$ thickness, deparaffinized in xylene and rehydrated with a graded ethanol series. The slides were boiled in $10 \mathrm{mmol} / \mathrm{l}$ citrate buffer ( $\mathrm{pH}$ 6.0) for $2.5 \mathrm{~min}$ at $100^{\circ} \mathrm{C}$ for antigen unmasking. The sections were immersed in $3 \% \mathrm{H}_{2} \mathrm{O}_{2}$ for 10 min to block the endogenous peroxidase and in goat serum blocking solution (cat. no. CW0130; CWbio Co., Ltd., Beijing, China) for $20 \mathrm{~min}$ to block non-specific antigens. The slides were incubated at room temperature for $2 \mathrm{~h}$ with primary antibodies for FAP- $\alpha$ (cat. no. ab53066, rabbit anti-human, 1:300; Abcam, Cambridge, UK), VEGF-A (cat. no. RAB-0157) and cluster of differentiation (CD)34 (kit-0004) (both from Fuzhou Maixin Biotech Co., Ltd., Fuzhou, China). The sections were washed with PBS and incubated in horseradish peroxidase-conjugated goat anti-rabbit/mouse immunoglobulin (Ig)G polymer (undiluted, cat. no. 9902; Fuzhou Maixin Biotech Co., Ltd.) at room temperature for $30 \mathrm{~min}$. Finally, slides were stained with 3,3'-diaminobenzidine and counterstained with $0.5 \%$ hematoxylin at room temperature. The assessment was performed by two independent pathologists who were blinded to clinical parameters and the clinical outcomes of the patients. The proportion score represented the estimated fraction of positively stained tumor cells: $0 \leq 25 \%, 26 \leq 1 \leq 50 \%$; $51 \leq 2 \leq 75 \%$; $3>75 \%$. The intensity score represented the estimated average staining intensity of positive tumor cells: 0 , Negative; 1 , weak; 2 , moderate; and 3, strong. The expression level of FAP- $\alpha$ and VEGF-A was evaluated using the product of proportion score and intensity score in 5 fields (x400, magnification) using a CX31 microscope (Olympus Corporation, Tokyo, Japan) and mean value was obtained ( $<4$ as low expression, $\geq 4$ as high expression). Microvessel density (MVD) was determined by
Table I. Overall survival differences between different groups.

\begin{tabular}{|c|c|}
\hline Comparisons between groups & P-value \\
\hline $\begin{array}{l}\text { FAP- } \alpha \text { low/VEGF-A high } \\
\text { vs. FAP- } \alpha \text { low/VEGF-A low }\end{array}$ & 0.457 \\
\hline $\begin{array}{l}\text { FAP- } \alpha \text { high/VEGF-A low } \\
\text { vs. FAP- } \alpha \text { high/VEGF-A high }\end{array}$ & 0.242 \\
\hline $\begin{array}{l}\text { FAP- } \alpha \text { low/VEGF-A high } \\
\text { vs. FAP- } \alpha \text { high/VEGF-A low }\end{array}$ & 0.833 \\
\hline $\begin{array}{l}\text { FAP- } \alpha \text { low/VEGF-A low } \\
\text { vs. FAP- } \alpha \text { high/VEGF-A high }\end{array}$ & $0.049^{\mathrm{a}}$ \\
\hline $\begin{array}{l}\text { FAP- } \alpha \text { low/VEGF-A high } \\
\text { vs. FAP- } \alpha \text { high/VEGF-A high }\end{array}$ & 0.308 \\
\hline $\begin{array}{l}\text { FAP- } \alpha \text { low/VEGF-A low } \\
\text { vs. FAP- } \alpha \text { high/VEGF-A low }\end{array}$ & 0.388 \\
\hline
\end{tabular}

${ }^{a} \mathrm{P}<0.05$ was considered to indicate a statistically significant difference. FAP- $\alpha$, fibroblast activation protein- $\alpha$; VEGF, vascular endothelial growth factor.

CD34 immunoreactivity and quantified as described previously (11).

Transfection. Plasmid $(0.5 \mu \mathrm{g})$ PcDNA3.1/FAP-vector (Shanghai GenePharma Co., Ltd., Shanghai, China) was transfected into SW1116 cells at $4 \times 10^{4}$ cells/well in a 6-well plate using Lipofectamine 2000 (Invitrogen; Thermo Fisher Scientific, Inc.) to upregulate FAP- $\alpha$ expression, and p-GPU6/FAP-short hairpin (sh)RNA (Shanghai GenePharma Co., Ltd.) was transfected into HT29 cells to silence FAP- $\alpha$ expression. Empty plasmids were used as the control. After $48 \mathrm{~h}$, the supernatant was collected as conditioned medium $(\mathrm{CM})$ and protein was extracted for further research.

Western blot analysis. Protein were extracted from cells using radioimmunoprecipitation lysis buffer containing $1 \%$ phenylmethanesulfonyl fluoride (Beyotime Institute of Biotechnology, Haimen, China) and total proteins were measured using a bicinchoninic acid kit (Pierce; Thermo Fisher Scientific, Inc.) according to the manufacturer's instructions. A total of $350 \mu \mathrm{g}$ total protein was loaded in each well of a $5 \%$ acrylamide gel and separated by a $10 \%$ separating gel prior to transfer onto a polyvinylidene difluoride membrane. After being blocked in 5\% non-fat milk (cat. no. 232100; BD Biosciences, Franklin Lakes, NJ, USA) at room temperature for $1 \mathrm{~h}$, the membrane was incubated with the primary antibodies at $4^{\circ} \mathrm{C}$ overnight and with peroxidase-linked goat anti-rabbit-IgG (cat. no. SA00001-2, 1:5,000; Proteintech Group Inc., Chicago, IL, USA) at room temperature for $1 \mathrm{~h}$. Signals were detected using enhanced chemiluminescence reagents (Pierce; Thermo Fisher Scientific, Inc.) and analyzed using Image-Pro Plus software (version 5.1; Media Cybernetics, Inc., Rockville, MD, USA). The primary antibodies were as follows: FAP- $\alpha$ (cat. no. ab53066, rabbit anti-human, 1:1,000; Epitomics, Burlingame, CA, USA); VEGF-A (cat. no. 19003-1-AP, 1:1,000; Proteintech Group, Inc.); VEGF-R2 (1:1,000; cat. 
A

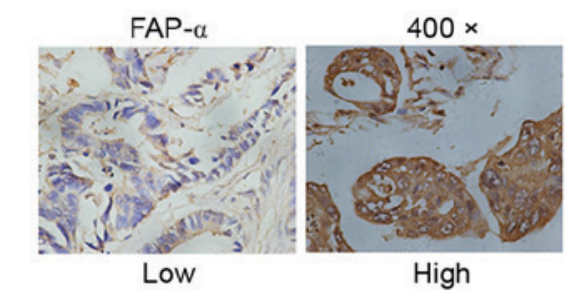

C

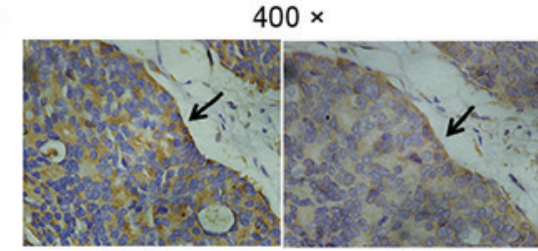

FAP $-\alpha$

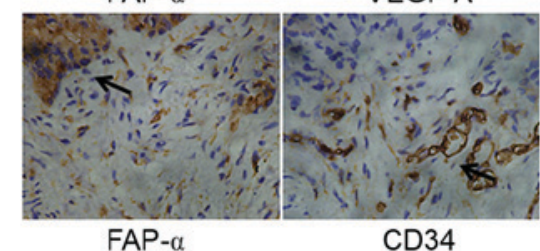

B

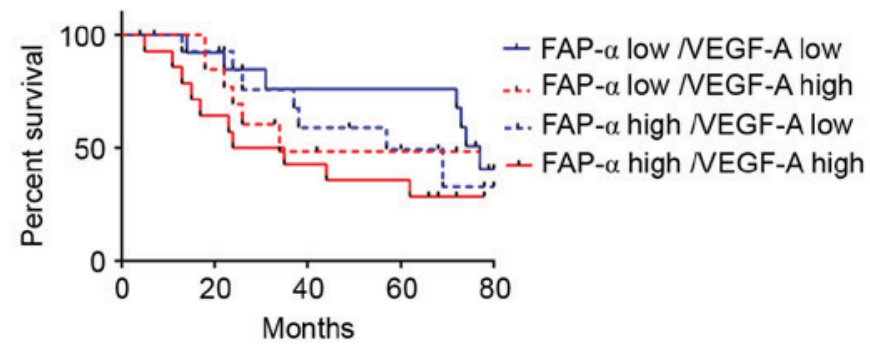

D

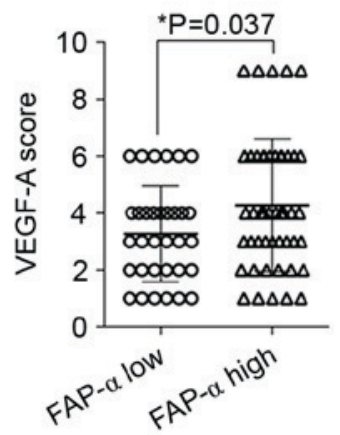

$E$

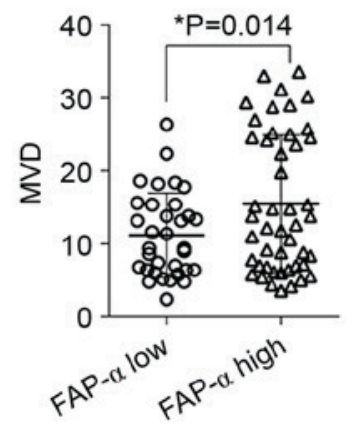

Figure 1. FAP- $\alpha$, VEGF-A and CD34 staining in CRC tissues. (A) Different expression levels of FAP- $\alpha$ stained via IHC in CRC tissues (magnification, $\mathrm{x} 400$ ), (B) IHC staining of VEGF-A and FAP- $\alpha$, and CD34 and FAP- $\alpha$ in serial slides (black arrows). (C) Statistical analysis of VEGF-A expression in the FAP- $\alpha$ low group and FAP- $\alpha$ high group. (D) Statistical analysis of MVD in the FAP- $\alpha$ low group and FAP- $\alpha$ high group. (E) Survival analysis of coexpression of FAP- $\alpha$ and VEGF-A using the Kaplan-Meier method. P $<0.05$ was considered to indicate a statistically significant difference. FAP, fibroblast activation protein; VEGF, vascular endothelial growth factor; CD, cluster of differentiation; CRC, colorectal cancer; IHC, immunohistochemistry; MVD, microvessel density.

no. ab2349; Abcam, Cambridge, MA, USA); phosphorylated-extracellular signal-regulated kinase (p-ERK)1/2 (cat. no. ab176660, 1:1,000; Epitomics; Abcam); ERK1/2 (cat. no. 9102, 1:1,000); phosphorylated-RAC- $\alpha$ serine/ threonine-protein kinase (cat. no. 13038, 1:1,000); and Akt (cat. no. 4685, 1:1,000) (all from Cell Signaling Technology, Inc.) and GAPDH (cat. no. 8727, 1:5,000; Wuhan Sanying Biotechnology, Wuhan, China).

ELISA analysis. VEGF-A in the supernatant was detected using a VEGF-A ELISA kit (cat. no. CSB-EL025833SH; Cusabio Biotech Co., Ltd., Wuhan, China), according to the manufacturer's instructions. The experiment was repeated at least three times.

MTT assay of HUVECs. Cells were counted and plated in 96-well plate in triplicate at $4 \times 10^{3}$ cells/well in $100 \mu 1$ medium. An MTT assay was performed dimethyl sulfoxide was used to dissolve formazan at 6 and $48 \mathrm{~h}$, according to the manufacturer's protocol (Beijing Solarbio Science and Technology Co., Ltd., Beijing, China). Optical density (OD) was measured at $490 \mathrm{~nm}$. The proliferation rate was calculated as the OD value at $48 \mathrm{~h} /$ the OD value at $6 \mathrm{~h}$. The experiment was repeated three times.

Statistical analysis. SPSS software version 13.0 (SPSS, Inc., Chicago, IL, USA) was used for statistical analysis. All data are expressed as the mean \pm standard deviation, and the differences between groups were analyzed using a one-way analysis of variance with Dunnett's post hoc test. Survival curves were drawn using the Kaplan-Meier method and compared using the log-rank test. $\mathrm{P}<0.05$ was considered to indicate a statistically significant difference.

\section{Results}

IHC staining of FAP- $\alpha, V E G F-A$ and $C D 34$ expression in $C R C$ tissues. As illustrated in Fig. 1A, FAP- $\alpha$ expression was stained by IHC in the cytoplasm of CRC cells, and sporadic staining was additionally identified in stromal cells. As presented in Fig. 1B, IHC staining of serial slides identified strong VEGF-A staining and more microvessels in FAP- $\alpha$ high expression tissues. Statistical analysis demonstrated that the general VEGF-A score (Fig. 1C) and MVD (Fig. 1D) in FAP- $\alpha$ high expression tissues was increased compared with FAP- $\alpha$ low expression tissues, with a statistically significant difference $(\mathrm{P}<0.05)$. Survival analysis demonstrated that patients whose tissue samples possessed high levels of FAP- $\alpha$ and VEGF-A demonstrated markedly worse outcomes in OS compared with those whose samples possessed low FAP- $\alpha$ or low VEGF-A expression levels, although the difference was only statistically significant when compared with samples of the low FAP- $\alpha$ and low VEGF-A expression group ( $\mathrm{P}=0.049$; Fig. 1E; Table I). All the results confirmed the association between FAP- $\alpha$ and angiogenesis at the tissue level and implied its important effects on the prognosis of patients with CRC.

FAP- $\alpha$ may regulate VEGF-A expression in CRC cells. To further confirm the proangiogenic roles of FAP- $\alpha$ at the cell level, FAP- $\alpha$ expression was altered via transfection in 

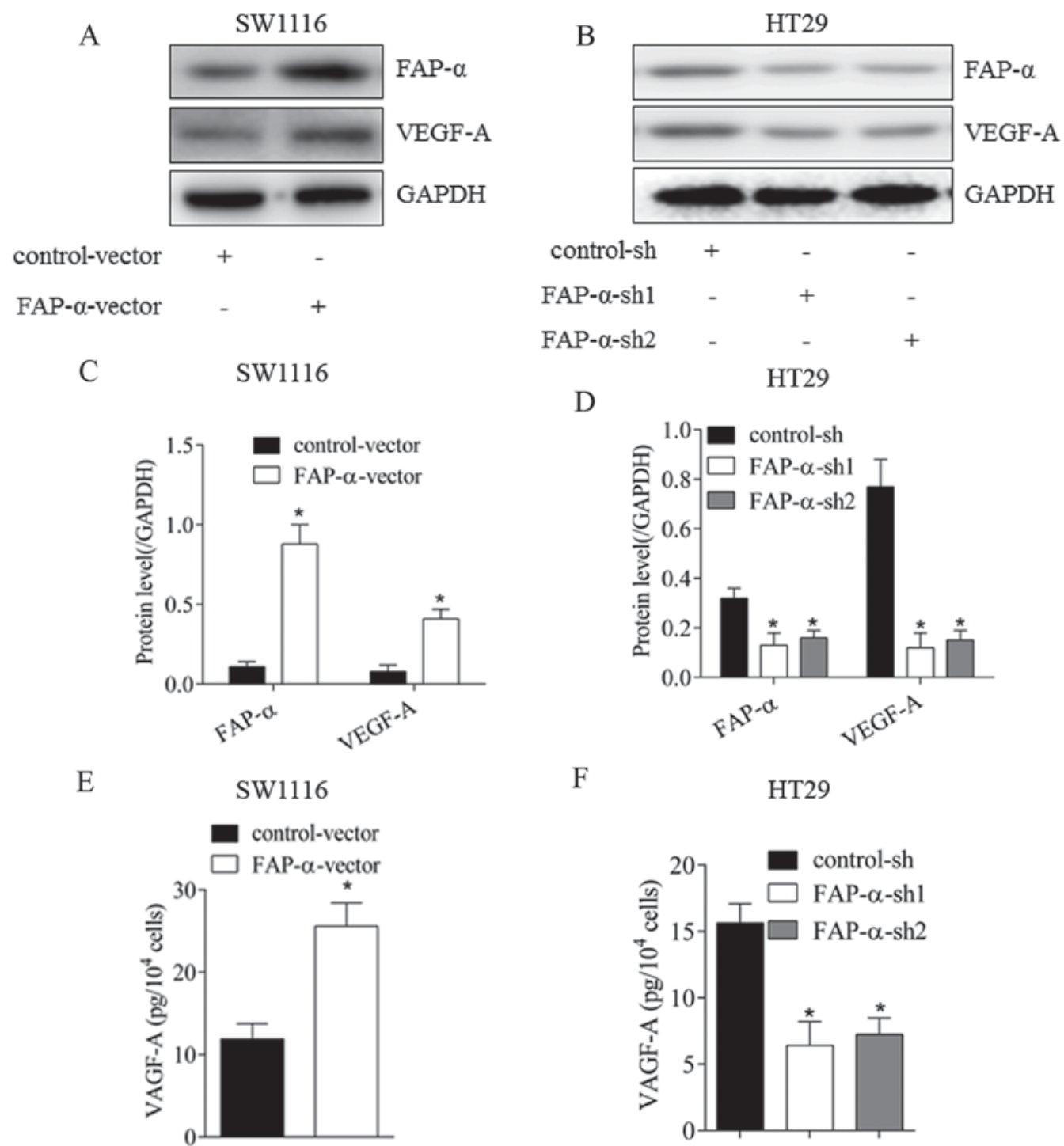

$\mathrm{D}$

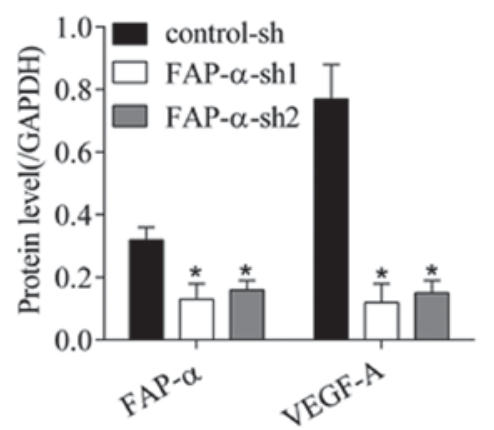

F

HT29

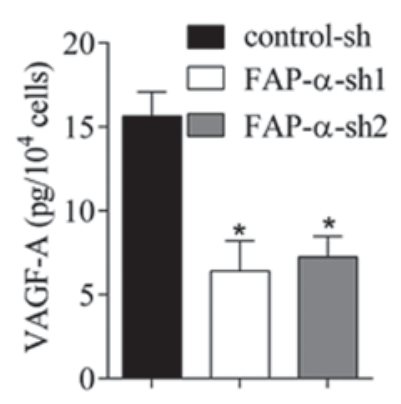

Figure 2. FAP- $\alpha$ regulates VEGF-A expression in CRC cell lines. (A) Following overexpression of FAP- $\alpha$ in SW1116 cells, changes of FAP- $\alpha$ and VEGF-A as demonstrated by western blot analysis. (B) Following silencing of FAP- $\alpha$ in HT29 cells, alterations in FAP- $\alpha$ and VEGF-A were demonstrated by western blot analysis. (C) Quantitative histogram for (A). (D) Quantitative histogram for (B). (E) Alterations in VEGF-A in the supernatant following overexpression of FAP- $\alpha$ in SW1116 cells. (F) Alterations in VEGF-A in the supernatant following silencing of FAP- $\alpha$ in HT29 cells. "P<0.05 vs. respective control. FAP, fibroblast activation protein; VEGF, vascular endothelial growth factor; CRC, colorectal cancer; sh, short hairpin.

CRC cells (Fig. 2). As illustrated in Fig. 2A and C, following upregulation of FAP- $\alpha$ in SW1116 cells, VEGF-A expression was significantly increased $(\mathrm{P}<0.05)$. The level of secreted VEGF-A in the supernatant was additionally elevated (Fig. 2E; P<0.05). Following silencing of FAP- $\alpha$ expression in HT29 cells, VEGF-A expression in the cytoplasm (Fig. 2B and $\mathrm{D} ; \mathrm{P}<0.05$ ) and supernatant (Fig. 2F; $\mathrm{P}<0.05$ ) declined significantly. These results verified that FAP- $\alpha$ may effectively promote the expression of the important proangiogenic factor VEGF-A in CRC cells.

FAP- $\alpha$ in CRC cells may activate HUVECs. To observe the effects of FAP- $\alpha$ shift on HUVECs, HUVECs were exposed to CM derived from SW1116 cells with overexpressed FAP- $\alpha$ (Fig. 3), and it was observed that the proliferation rate of HUVECs increased significantly from $0.9 \pm 0.11$ to $1.54 \pm 0.07$ (Fig. 3A). VEGF-R2, p-ERK and p-Akt were significantly upregulated (Fig. 3C). Conversely, following treatment using
CM derived from HT29 cells with silenced FAP- $\alpha$, the proliferation rate of HUVECs declined significantly from $0.96 \pm 0.09$ to $0.42 \pm 0.04$ or $0.48 \pm 0.04$ (Fig. 3B). These results demonstrated the activating roles of FAP- $\alpha$ in CRC cells on HUVECs.

FAP- $\alpha$ regulates VEGF-A expression via Akt and ERK signaling pathways. To further study the potential molecular mechanism responsible for the proangiogenic effects of FAP- $\alpha$, p-ERK and p-Akt were selected as candidate signal targets. Western blot analysis identified that FAP- $\alpha$ overexpression in SW1116 cells was able to activate the phosphorylation of ERK and Akt (Fig. 4A). Phosphorylation of ERK and Akt was additionally inhibited following FAP- $\alpha$ silencing in HT29 cells (Fig. 4B). To further confirm whether p-ERK and p-Akt are involved in the regulation of VEGF-A expression, western blot analysis was performed and it was identified that the Akt inhibitor LY294002 (Fig. 4C) and ERK inhibitor 
A

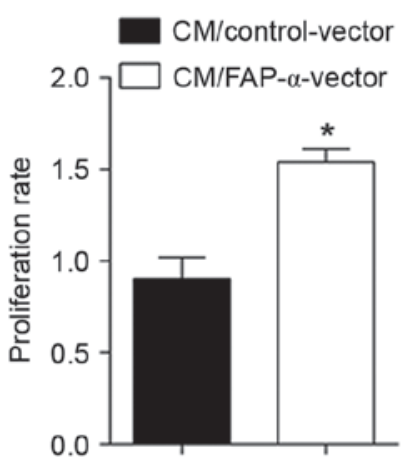

B

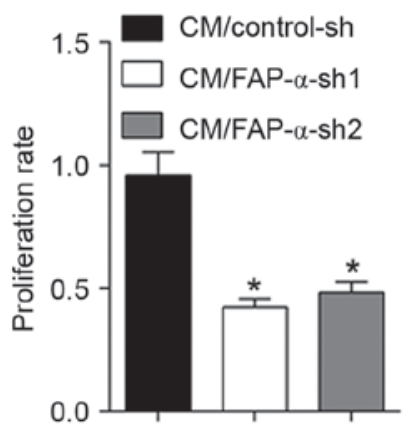

C

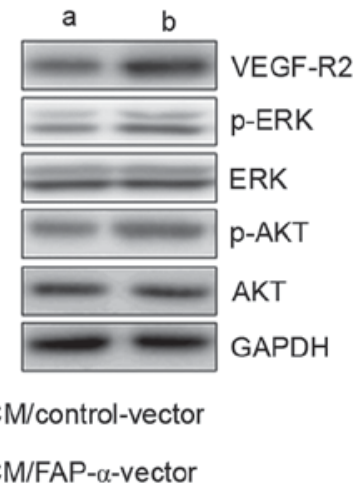

Figure 3. FAP- $\alpha$ activates HUVECs. (A) Alteration in the proliferation rate of HUVECs following treatment with CM from SW1116 cells overexpressing FAP- $\alpha$. (B) Alteration in the proliferation rate of HUVECs following treatment with CM from HT29 cells with silenced FAP- $\alpha$. (C) Alteration in VEGF-R2 expression, p-ERK and p-Akt in HUVECs following treatment with CM from SW1116 cells overexpressing FAP- $\alpha$. "P $<0.05$ vs. control. FAP, fibroblast activation protein; HUVECs, human umbilical vein endothelial cells; CM, conditioned medium VEGF, vascular endothelial growth factor; p, phosphorylated; ERK, extracellular signal-regulated kinase; Akt, RAC- $\alpha$ serine/threonine-protein kinase; sh, short hairpin.

A

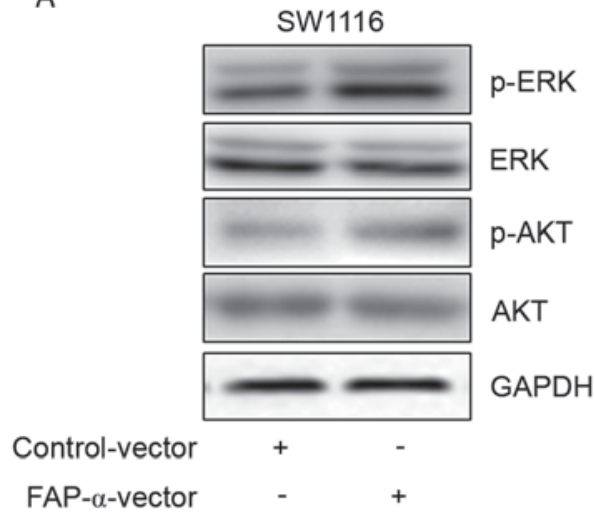

C

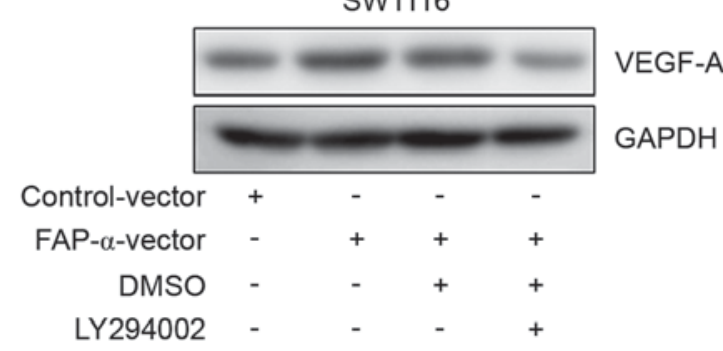

B

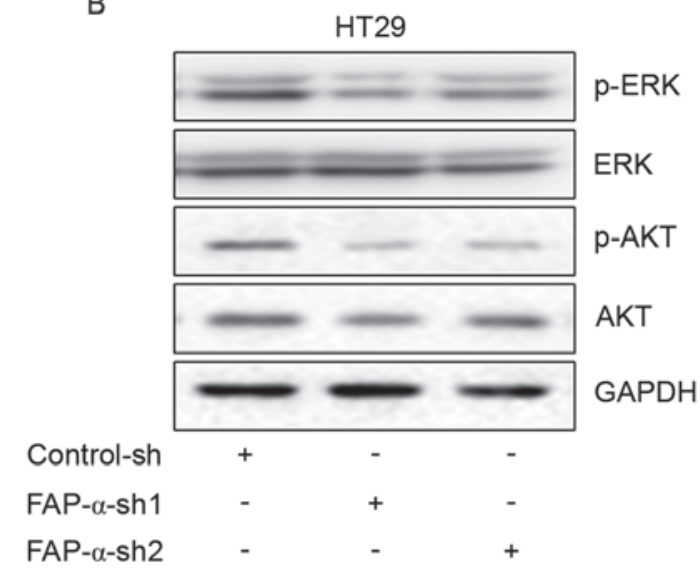

D

SW1116

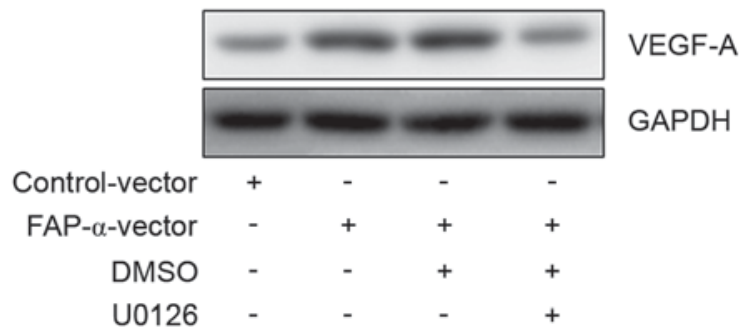

Figure 4. Akt and ERK signaling pathways are required for VEGF-A upregulation induced by FAP- $\alpha$. (A) Following overexpression of FAP- $\alpha$ in SW1116 cells, alterations in p-ERK and p-Akt expression were demonstrated by western blot analysis. (B) Following silencing of FAP- $\alpha$ in HT29 cells, alterations in p-ERK and p-Akt expression demonstrated by western blot analysis. (C) Alterations in VEGF-A expression in FAP- $\alpha$-overexpressing SW1116 cells following treatment with LY294002. (D) Alterations in VEGF-A expression in FAP- $\alpha$-overexpressing SW1116 cells following treatment with U0126. Akt, RAC- $\alpha$ serine/threonine-protein kinase; ERK, extracellular signal-regulated kinase; VEGF, vascular endothelial growth factor; FAP, fibroblast activation protein; p, phosphorylated; DMSO, dimethyl sulfoxide; sh, short hairpin.

U0126 (Fig. 4D) significantly abrogated VEGF-A upregulation induced by FAP- $\alpha$. These results demonstrated that Akt and ERK were involved in the proangiogenic effects of FAP- $\alpha$.

\section{Discussion}

FAP- $\alpha$ is an integral membrane serine peptidase and tumor stromal FAP- $\alpha$ has been considered to be an important participant in tumor onset and progression, and even prognosis, through enzymatic and non-enzymatic functions $(12,13)$. Wikberg et al (14) reported that stromal FAP- $\alpha$ in tumor boundaries was not associated with patient prognosis, while high stromal FAP- $\alpha$ expression in the center of a tumor was positively correlated with poor prognosis. In ovarian cancer, stromal FAP- $\alpha$ was positively correlated with lymph node and omental metastasis, in addition to elevated lymphatic density (15). Based 
on the important roles of stromal FAP- $\alpha$ in tumor progression, FAP- $\alpha$ in tumor cells has attracted increasing interest and its expression has been detected in a number of types of epithelial tumor cells, including breast cancer (16), pancreatic adenocarcinoma (17), gastric cancer (18), oral squamous cell carcinoma (19), ovarian cancer (20), cervical cancer (21) and CRC (10). Jia et al (22) reported that FAP- $\alpha$ was significantly associated with poor outcome in patients with breast cancer, and promoted the proliferation and inhibited the migration of breast cancer cells. Lai et al (23) identified that silencing of FAP- $\alpha$ in the ovarian cancer cell line SKOV3 inhibited tumor growth in vivo. Shi et al (17) reported that increased FAP- $\alpha$ expression in pancreatic adenocarcinoma cells was associated with tumor size, fibrotic focus, perineural invasion and a worse clinical outcome. The present study detected FAP- $\alpha$ expression at the tissue level via IHC and at the cell level by western blotting, and further verified its presence in CRC cells. IHC staining identified that FAP- $\alpha$ in CRC cells was positively associated with MVD and VEGF-A expression, implying its proangiogenic effects for the first time, to the best of the authors' knowledge. VEGF-A is the most important regulatory factor in tumor angiogenesis, and altered expression of VEGF-A has been reported to be associated with a poor prognosis in various types of human cancer $(24,25)$. The positive association of FAP- $\alpha$ and VEGF-A suggested that FAP- $\alpha$ may function in angiogenesis by regulating VEGF-A expression. To test this hypothesis, FAP- $\alpha$ expression was altered by transfection and it was identified that VEGF-A expression exhibited the same alterations in the cytoplasm and supernatant. Patients with double high expression of FAP- $\alpha$ and VEGF-A exhibited the worst prognosis, compared with the high FAP- $\alpha$ only or high VEGF-A only groups, implying that FAP- $\alpha$ alone or VEGF-A alone may not exert significant effects on prognosis, although their coexpression posessed notable clinical significance. This was not consistent with a previous study (24), possibly due to different samples or the limited number of cases in the present study.

VEGFR-2 is an essential mediator of VEGF-initiated angiogenesis and serves crucial roles in regulating multiple signaling pathways in endothelial cells which may regulate core angiogenic responses, including proliferation, migration and tube formation abilities $(26,27)$. The Akt and ERK signaling pathways are crucial participants in maintaining the different biological behaviors of different cells including proliferation, migration, differentiation, drug resistance, apoptosis and phenotype maintenance (28-30). To detect the direct effects of FAP- $\alpha$ alterations on HUVECs, HUVECs were treated using CM from SW1116 cells with FAP- $\alpha$ overexpression, and it was identified that the expression of VEGF-R2, p-Akt and p-ERK all increased significantly, as did proliferation ability. CM from HT29 cells with silenced FAP- $\alpha$ expression exerted the opposite effect on the proliferation ability of HUVECs. This further confirmed the proangiogenic function of FAP- $\alpha$ in CRC cells. p-Akt and p-ERK in SW1116 cells and HT29 cells were significantly affected by FAP- $\alpha$ expression. The p-Akt inhibitor LY294002 and the p-ERK inhibitor U0126 were able to inhibit VEGF-A upregulation induced by FAP- $\alpha$ overexpression. This implied that endogenous FAP- $\alpha$ in CRC cells may regulate the VEGF-A expression of CRC cells and HUVEC activation via the Akt and ERK signaling pathways.
In conclusion, the present study provided further evidence of the presence of FAP- $\alpha$ in CRC cells, and additionally demonstrated that FAP- $\alpha$ in CRC cells was able to promote angiogenesis via the Akt and ERK signaling pathways. This provided novel knowledge about the functions of endogenous FAP- $\alpha$ in tumor cells and supplied further evidence for treating FAP- $\alpha$ as a target in therapy.

\section{References}

1. Hubbard JM: Management of colorectal cancer in older adults. Clin Geriatr Med 32: 97-111, 2016

2. Arcaroli JJ, Tai WM, McWilliams R, Bagby S, Blatchford PJ, Varella-Garcia M, Purkey A, Quackenbush KS, Song EK, Pitts TM, et al: A NOTCH1 gene copy number gain is a prognostic indicator of worse survival and a predictive biomarker to a Notch1 targeting antibody in colorectal cancer. Int J Cancer 138: 195-205, 2016

3. Dickinson KJ and Blackmon SH: Results of pulmonary resection: Colorectal carcinoma. Thorac Surg Clin 26: 41-47, 2016.

4. Kuijer A, Furnée EJ and Smakman N: Combined surgery for primary colorectal cancer and synchronous pulmonary metastasis: A pilot experience in two patients. Eur J Gastroenterol Hepatol 28: 15-19, 2016.

5. Li J, Zhang Y, Zhao Q, Wang J and He X: MicroRNA-10a influences osteoblast differentiation and angiogenesis by regulating $\beta$-catenin expression. Cell Physiol Biochem 37: 2194-2208, 2015.

6. Salem A and O'Connor JP: Assessment of tumor angiogenesis: Dynamic contrast-enhanced MR imaging and beyond. Magn Reson Imaging Clin N Am 24: 45-56, 2016.

7. Mhawech-Fauceglia P, Yan L, Sharifian M, Ren X, Liu S, Kim G, Gayther SA, Pejovic T and Lawrenson K: Stromal expression of fibroblast activation protein alpha (FAP) predicts platinum resistance and shorter recurrence in patients with epithelial ovarian cancer. Cancer Microenviron 8: 23-31, 2015.

8. Huang Y, Wang S and Kelly T: Seprase promotes rapid tumor growth and increased microvessel density in a mouse model of human breast cancer. Cancer Res 64: 2712-2716, 2004.

9. Cheng JD, Dunbrack RL Jr, Valianou M, Rogatko A, Alpaugh RK and Weiner LM: Promotion of tumor growth by murine fibroblast activation protein, a serine protease, in an animal model. Cancer Res 62: 4767-4772, 2002.

10. Iwasa S, Jin X, Okada K, Mitsumata M and Ooi A: Increased expression of seprase, a membrane-type serine protease, is associated with lymph node metastasis in human colorectal cancer. Cancer Lett 199: 91-98, 2003.

11. Vermeulen PB, Gasparini G, Fox SB, Colpaert C, Marson LP, Gion M, Beliën JA, de Waal RM, Van Marck E, Magnani E, et al: Second international consensus on the methodology and criteria of evaluation of angiogenesis quantification in solid human tumours. Eur J Cancer 38: 1564-1579, 2002.

12. Kelly T1, Huang Y, Simms AE and Mazur A: Fibroblast activation protein- $\alpha$ : A key modulator of the microenvironment in multiple pathologies. Int Rev Cell Mol Biol 297: 83-116, 2012.

13. Huang Y, Simms AE, Mazur A, Wang S, León NR, Jones B, Aziz N and Kelly T: Fibroblast activation protein- $\alpha$ promotes tumor growth and invasion of breast cancer cells through non-enzymatic functions. Clin Exp Metastasis 28: 567-579, 2011.

14. Wikberg ML, Edin S, Lundberg IV, Van Guelpen B, Dahlin AM, Rutegård J, Stenling R, Oberg A and Palmqvist R: High intratumoral expression of fibroblast activation protein (FAP) in colon cancer is associated with poorer patient prognosis. Tumour Biol 34: 1013-1020, 2013

15. Schauer IG, Sood AK, Mok S and Liu J: Cancer-associated fibroblasts and their putative role in potentiating the initiation and development of epithelial ovarian cancer. Neoplasia 13: 393-405, 2011.

16. Kelly T, Kechelava S, Rozypal TL, West KW and Korourian S: Seprase, a membrane-bound protease, is overexpressed by invasive ductal carcinoma cells of human breast cancers. Mod Pathol 11: 855-863, 1998.

17. Shi M, Yu DH, Chen Y, Zhao CY, Zhang J, Liu QH, Ni CR and Zhu MH: Expression of fibroblast activation protein in human pancreatic adenocarcinoma and its clinicopathological significance. World J Gastroenterol 18: 840-846, 2012. 
18. Mori Y, Kono K, Matsumoto Y, Fujii H, Yamane T, Mitsumata M and Chen WT: The expression of a type II transmembrane serine protease (Seprase) in human gastric carcinoma. Oncology 67: 411-419, 2004

19. Wang H, Wu Q, Liu Z, Luo X, Fan Y, Liu Y, Zhang Y, Hua S $\mathrm{Fu} \mathrm{Q}$, Zhao M, et al: Downregulation of FAP suppresses cell proliferation and metastasis through PTEN/PI3K/AKT and Ras-ERK signaling in oral squamous cell carcinoma. Cell Death Dis 5: e1155, 2014.

20. Kennedy A, Dong H, Chen D and Chen WT: Elevation of seprase expression and promotion of an invasive phenotype by collagenous matrices in ovarian tumor cells. Int J Cancer 124: 27-35, 2009.

21. Jin X, Iwasa S, Okada K, Mitsumata M and Ooi A: Expression patterns of seprase, a membrane serine protease, in cervical carcinoma and cervical intraepithelial neoplasm. Anticancer Res 23: 3195-3198, 2003.

22. Jia J, Martin TA and Jiang WG: FAP- $\alpha$ (fibroblast activation protein- $\alpha$ ) is involved in the control of human breast cancer cell line growth and motility via the FAK pathway. BMC Cell Biol 15: 16, 2014.

23. Lai D, Ma L and Wang F: Fibroblast activation protein regulates tumor-associated fibroblasts and epithelial ovarian cancer cells. Int J Oncol 41: 541-550, 2012.

24. Lin J, Yun D, Tao Li, Yalei L, Yudong W, Yan Z, Xinliang Z and Wei L: Differential expression of vascular endothelial growth factor-A, $-\mathrm{C}$ and -D for the diagnosis and prognosis of cancer patients with malignant effusions. Oncol Lett 10: 667-674, 2015.
25. Zhao $\mathrm{H}, \mathrm{Wu} \mathrm{Y}$, Chen $\mathrm{Y}$ and Liu $\mathrm{H}$ : Clinical significance of hypoxia-inducible factor 1 and VEGF-A in osteosarcoma. Int J Clin Oncol 20: 1233-1243, 2015.

26. Gheorghescu AK, Tywoniuk B, Duess J, Buchete NV and Thompson J: Exposure of chick embryos to cadmium changes the extra-embryonic vascular branching pattern and alters expression of VEGF-A and VEGF-R2. Toxicol Appl Pharmacol 289: 79-88, 2015.

27. Adeoye OO, Bouthors V, Hubbell MC, Williams JM and Pearce WJ: VEGF receptors mediate hypoxic remodeling of adult ovine carotid arteries. J Appl Physiol (1985) 117: 777-787, 2014.

28. D'Amico MA, Ghinassi B, Izzicupo P, Di Ruscio A and Di Baldassarre A: IL- 6 activates PI3K and PKC $\zeta$ signaling and determines cardiac differentiation in rat embryonic $\mathrm{H} 9 \mathrm{c} 2$ cells. J Cell Physiol 231: 576-586, 2016.

29. Ge C, Cawthorn WP, Li Y, Zhao G, Macdougald OA and Franceschi RT: Reciprocal control of osteogenic and adipogenic differentiation by ERK/MAP kinase phosphorylation of Runx2 and PPAR $\gamma$ transcription factors. J Cell Physiol 231: 587-596, 2016.

30. Ishii $\mathrm{M}$, Nakahara $\mathrm{T}$, Ikeuchi $\mathrm{S}$ and Nishimura $\mathrm{M}$ : $\beta$-amyrin induces angiogenesis in vascular endothelial cells through the Akt/endothelial nitric oxide synthase signaling pathway. Biochem Biophys Res Commun 467: 676-682, 2015. 